\title{
FALCONER DISTANCE PROBLEM, ADDITIVE ENERGY AND CARTESIAN PRODUCTS
}

\author{
Alex Iosevich and Bochen Liu \\ University of Rochester, Department of Mathematics \\ RC Box 270138, Rochester, NY 14627, U.S.A.; iosevich@math.rochester.edu \\ University of Rochester, Department of Mathematics \\ RC Box 270138, Rochester, NY 14627, U.S.A.; bochen.liu@rochester.edu
}

\begin{abstract}
A celebrated result due to Wolff says if $E$ is a compact subset of $\mathbf{R}^{2}$, then the Lebesgue measure of the distance set $\Delta(E)=\{|x-y|: x, y \in E\}$ is positive if the Hausdorff dimension of $E$ is greater than $\frac{4}{3}$. In this paper we improve the $\frac{4}{3}$ barrier by a small exponent for Cartesian products. In higher dimensions, also in the context of Cartesian products, we reduce Erdogan's $\frac{d}{2}+\frac{1}{3}$ exponent to $\frac{d^{2}}{2 d-1}$. The proof uses a combination of Fourier analysis and additive comibinatorics.
\end{abstract}

\section{Introduction}

The Falconer distance conjecture [3] says that if the Hausdorff dimension of $E \subset \mathbf{R}^{d}, d \geq 2$, is greater than $\frac{d}{2}$, then the Lebesgue measure of the distance set $\Delta(E)=\{|x-y|: x, y \in E\}$ is positive.

The best known results are due to Wolff [7] in two dimensions and Erdogan [2] in higher dimensions. They proved that the Lebesgue measure of $\Delta(E)$ is positive if the Hausdorff dimension of $E$ is greater than $\frac{d}{2}+\frac{1}{3}$. This was accomplished by showing that if $s \in\left(\frac{d}{2}, \frac{d+2}{2}\right)$ is the Hausdorff dimension of $E$ and $\mu$ is a Frostman measure on $E$ which has finite $(s-\epsilon)$-energy $I_{s-\epsilon}(\mu)$ for all $\epsilon>0$, then for all $\epsilon>0$,

$$
\int_{S^{d-1}}|\widehat{\mu}(t \omega)|^{2} d \omega \leq C t^{-\frac{d+2 s-2}{4}+\epsilon}
$$

In particular, in the two-dimensional case, which is the focus of this paper, the estimate takes the form

$$
\int_{S^{1}}|\widehat{\mu}(t \omega)|^{2} d \omega \leq C t^{-\frac{s}{2}+\epsilon}
$$

This estimate is then plugged into the Mattila integral,

$$
\mathcal{M}(\mu)=\int_{1}^{\infty}\left(\int_{S^{d-1}}|\widehat{\mu}(t \omega)|^{2} d \omega\right)^{2} t^{d-1} d t,
$$

the most effective tool developed so far for the study of the Falconer distance problem.

Mattila proved in [5] that if $E$ is a compact set of Hausdorff dimension $s>\frac{d}{2}$ and $\mu$ is Borel measure supported on $E$ such that $\mathcal{M}(\mu)<\infty$, then the Lebesgue measure of $\Delta(E)$ is positive. For discussion about other versions of Mattila integrals, see [4].

Our results are the following.

doi:10.5186/aasfm.2016.4135

2010 Mathematics Subject Classification: Primary 28A75, 52C10.

Key words: Distance problem, Cartesian products, additive energy, Ahlfors-David regular. 
Theorem 1.1. Let $E=A \times B$, where $A$ and $B$ are compact subsets of $\mathbf{R}$ with positive $s_{A}, s_{B}$-dimensional Hausdorff measure, respectively. If $s_{A}+s_{B}+\max \left(s_{A}, s_{B}\right)$ $>2$, the Lebesgue measure of $\Delta(E)$ is positive. In particular, if $\operatorname{dim}_{\mathcal{H}}(E)=\operatorname{dim}_{\mathcal{H}}(A)+$ $\operatorname{dim}_{\mathcal{H}}(B)$ and $\operatorname{dim}_{\mathcal{H}}(A) \neq \operatorname{dim}_{\mathcal{H}}(B), \operatorname{dim}_{\mathcal{H}}(E)>\frac{4}{3}-\frac{\left|\operatorname{dim}_{\mathcal{H}}(A)-\operatorname{dim}_{\mathcal{H}}(B)\right|}{3} \operatorname{implies} \Delta(E)$ has positive Lebesgue measure.

To state the result in the case $\operatorname{dim}_{\mathcal{H}}(A)=\operatorname{dim}_{\mathcal{H}}(B)$, we need the following definition.

Definition 1.2. Let $A$ be a compact subset of $\mathbf{R}^{d}$ of Hausdorff dimension $s_{A}$. We say $A$ is Ahlfors-David regular if there exists a Radon measure $\nu_{A}$ on $A$ and a constant $0<C_{\nu_{A}}<\infty$ such that

$$
C_{\nu_{A}}^{-1} r^{s_{A}}<\nu_{A}(B(x, r))<C_{\nu_{A}} r^{s_{A}}, \forall x \in A, 0<r<1 .
$$

Theorem 1.3. Suppose $E=A \times B, s_{A}=s_{B}=\alpha$ and $A$ is Ahlfors-David regular with $\nu_{A}, C_{\nu_{A}}$ such that (1.4) holds. Then there exists $\delta=\delta\left(C_{\nu_{A}}\right)>0$ such that whenever $\alpha>\frac{2}{3}-\delta$, the Lebesgue measure of $\Delta(E)$ is positive.

We also obtain an improvement of Erdogan's $\frac{d}{2}+\frac{1}{3}$ exponent in higher dimension for Cartesian products.

Theorem 1.4. Suppose that $E$ is a compact subset of $\mathbf{R}^{d}$ of the form $A_{1} \times$ $A_{2} \times \cdots \times A_{d}$, where $A_{j} \subset \mathbf{R}$ has positive $s_{j}$-dimensional Hausdorff measure for all $1 \leq j \leq d$. Suppose that $\sum_{j=1}^{d} s_{j}>\frac{d^{2}}{2 d-1}$. Then the Lebesgue measure of $\Delta(E)$ is positive.

1.1. Outline of the proof of Theorems 1.1, 1.3 and 1.4. Our argument consists of three basic steps.

- We first establish Theorem 1.1 which is accomplished using the imbalance inherent in the structure of the Mattila integral.

- The improvement of the $\frac{d}{2}+\frac{1}{3}$ exponent for Cartesian products in higher dimensions (Theorem 1.4) is accomplished in the same way regardless of whether the Hausdorff dimension of the fibers is the same.

- In the case when $\operatorname{dim}_{\mathcal{H}}(A)=\operatorname{dim}_{\mathcal{H}}(B)$, we use a recent result due to Dyatlov and Zahl [1] to show that when $A$ is Ahlfors-David regular, the additive energy of $A$ at scale $t^{-1}$,

$$
\nu_{A}^{4}\left\{\left(a_{1}, a_{2}, a_{3}, a_{4}\right):\left|\left(a_{1}+a_{2}\right)-\left(a_{3}+a_{4}\right)\right| \leq t^{-1}\right\},
$$

where $\nu_{A}$ is a Frostman measure on $A$, satisfies a better than trivial estimate, namely $C t^{-\operatorname{dim}_{\mathcal{H}}(A)-\delta}$ for some $\delta>0$, and then show that this leads to a slightly better exponent than $\frac{4}{3}$.

Acknowledgements. The authors wish to thank Josh Zahl and the anonymous referee for several useful suggestions.

\section{Proof of Theorem 1.1}

We shall repeatedly use the following simple estimate.

Lemma 2.1. (Solid Average) Suppose that $\nu$ is a compactly supported Borel measure on $\mathbf{R}^{d}$ such that $\nu(B(x, r)) \leq C r^{\alpha}$ for all $x \in \mathbf{R}^{d}$. Then for any bounded rectangle $R$,

$$
\int_{R}|\widehat{\nu}(t u)|^{2} d u \leq C_{R} t^{-\alpha} .
$$


To prove the lemma observe that the left hand side is

$$
\leq \int|\widehat{\nu}(t u)|^{2} \widehat{\psi}(u) d u
$$

where $\psi$ is a suitably chosen smooth compactly supported function. This expression equals

$$
\iiint e^{2 \pi i(x-y) \cdot t u} \widehat{\psi}(u) d u d \nu(x) d \nu(y)=\iint \psi(t(x-y)) d \nu(x) d \nu(y) \leq C_{R} t^{-\alpha}
$$

by assumption. This completes the proof of the lemma.

We now parameterize the upper semi-circle $S_{1}^{+}$in the form

$$
\left\{\left(u, \sqrt{1-u^{2}}\right):-1 \leq u \leq 1\right\} \text {. }
$$

The argument shall be carried out for this parameterization as the proof for the lower semi-circle is identical.

Let $d \mu(x)=d \nu_{A}\left(x_{1}\right) d \nu_{B}\left(x_{2}\right)$, where $\nu_{A}, \nu_{B}$ are Frostman probability measures on $A$ and $B$, respectively such that

$$
\nu_{A}(B(x, r)) \leq C r^{s_{A}}, \quad \nu_{B}(B(x, r)) \leq C r^{s_{B}} .
$$

Assume without loss of generality that $s_{A} \geq s_{B}$. Also assume $E$ is not a point mass, which implies that either

$$
\exists a \in \mathbf{R}, \mu\left(\left\{\left(x_{1}, x_{2}\right): x_{1}>a\right\}\right), \mu\left(\left\{\left(x_{1}, x_{2}\right): x_{1}<a\right\}\right)>0,
$$

or

$$
\exists b \in \mathbf{R}, \mu\left(\left\{\left(x_{1}, x_{2}\right): x_{2}>b\right\}\right), \mu\left(\left\{\left(x_{1}, x_{2}\right): x_{2}<b\right\}\right)>0 .
$$

Without loss of generality, we may assume $\mu\left(\left\{\left(x_{1}, x_{2}\right): x_{2}>b\right\}\right), \mu\left(\left\{\left(x_{1}, x_{2}\right): x_{2}<\right.\right.$ $b\})>0$ for some $b \in \mathbf{R}$. It follows that

$$
\iint \frac{\left|x_{2}-y_{2}\right|}{|x-y|} d \mu(x) d \mu(y)>0 \text {. }
$$

Let $\omega=(\cos (\theta), \sin (\theta))$. Consider the modified Mattila integral

$$
\int\left(\int_{S^{1}}|\widehat{\mu}(t \omega)|^{2}|\sin (\theta)| d \omega\right)^{2} t d t .
$$

Lemma 2.2. Suppose (2.1) holds. Then the finiteness of the integral (2.2) implies that the Lebesgue measure of the distance set is positive.

Proof. To prove this lemma, one simply replaces the distance measure in the derivation of the Mattila integral in [7], given by the relation

$$
\int f(t) d \nu_{0}^{*}(t)=\iint f(|x-y|) d \mu(x) d \mu(y)
$$

by a slightly modified distance measure given by

$$
\int f(t) d \nu_{0}(t)=\iint f(|x-y|) \frac{\left|x_{2}-y_{2}\right|}{|x-y|} d \mu(x) d \mu(y) .
$$

As in [7], define

$$
d \nu(t)=e^{i \frac{\pi}{4}} t^{-\frac{1}{2}} d \nu_{0}(t)+e^{-i \frac{\pi}{4}}|t|^{-\frac{1}{2}} d \nu_{0}(-t)
$$


and it follows that

$$
\widehat{\nu}(t)=\iint|x-y|^{-\frac{1}{2}} \cos \left(2 \pi\left(t|x-y|-\frac{1}{8}\right)\right) \frac{\left|x_{2}-y_{2}\right|}{|x-y|} d \mu(x) d \mu(y) .
$$

On the other hand,

$$
\int|\widehat{\mu}(t \omega)|^{2}|\sin \theta| d \theta=\iint\left(\int e^{2 \pi i(x-y) \cdot(t \omega)}|\sin \theta| d \theta\right) d \mu(x) d \mu(y) .
$$

Let $\theta_{x-y}$ be the angle between the vector $x-y$ and the $x$-axis. Then $\left|\sin \theta_{x-y}\right|=$ $\frac{\left|x_{2}-y_{2}\right|}{|x-y|}$. We may assume $s$, the Hausdorff dimension of $E$, is not greater than $\frac{3}{2}$. By stationary phase(see, e.g. [8] for details),

$$
\begin{aligned}
& \int e^{2 \pi i(x-y) \cdot(t \omega)}|\sin \theta| d \theta \\
& =2(|t||x-y|)^{-\frac{1}{2}} \cos \left(2 \pi\left(t|x-y|-\frac{1}{8}\right)\right)\left|\sin \theta_{x-y}\right|+O\left((t|x-y|)^{-\frac{3}{2}}\right) \\
& =2(|t||x-y|)^{-\frac{1}{2}} \cos \left(2 \pi\left(t|x-y|-\frac{1}{8}\right)\right) \frac{\left|x_{2}-y_{2}\right|}{|x-y|}+O\left((t|x-y|)^{-s+\epsilon}\right) .
\end{aligned}
$$

Putting (2.3), (2.4), (2.5) together, one can see

$$
\begin{aligned}
\|\widehat{\nu}\|_{2}^{2} & =\int_{|t| \leq 1}|\widehat{\nu}(t)|^{2} d t+\int_{|t| \geq 1}|\widehat{\nu}(t)|^{2} d t \\
& \leq 1+\int_{1}^{\infty}\left(\int|\widehat{\mu}(t \omega)|^{2}|\sin \theta| d \theta\right)^{2} t d t+C I_{s-\epsilon}(\mu)
\end{aligned}
$$

which proves the lemma since $I_{s-\epsilon}(\mu)<\infty$.

We now proceed with the estimation of (2.2). It follows that

$$
\int_{S^{1}}|\widehat{\mu}(t \omega)|^{2}|\sin (\theta)| d \omega
$$

is bounded by the sum of two terms of the form

$$
\int_{-1}^{1}\left|\widehat{\nu}_{A}(t u)\right|^{2}\left|\widehat{\nu}_{B}\left( \pm t \sqrt{1-u^{2}}\right)\right|^{2} d u \leq \int_{-1}^{1}\left|\widehat{\nu}_{A}(t u)\right|^{2} d u \leq C t^{-s_{A}}
$$

by Lemma 2.1. Plugging (2.6) into the modified Mattila integral (2.2) we see that

$$
\begin{aligned}
\mathcal{M}(\mu) & \leq C \iint_{S^{1}}|\widehat{\mu}(t \omega)|^{2} t \cdot t^{-s_{A}} d \omega d t=C \int|\widehat{\mu}(\xi)|^{2}|\xi|^{-s_{A}} d \xi \\
& =C^{\prime} \iint|x-y|^{-2+s_{A}} d \mu(x) d \mu(y)
\end{aligned}
$$

and this energy integral (see e.g. [8] or [6]) is finite if

$$
s_{A}+s_{B}>2-s_{A}
$$

as desired. 


\section{Proof of Theorem $\mathbf{1 . 3}$}

We improve the upper bound of (1.2) to prove the theorem. More precisely, under the assumptions of Theorem 1.3, there exists $\delta=\delta\left(C_{\nu_{A}}\right)>0$ such that

$$
\int_{S^{1}}|\hat{\mu}(t \omega)|^{2} d \omega \lesssim t^{-\alpha-\delta}
$$

where $\mu=\nu_{A} \times \nu_{B}$. First we deal with the case when $\theta$ is close to 0 . We have

$$
\int_{0}^{\delta}\left|\widehat{\nu}_{A}(t \cos (\theta))\right|^{2}\left|\widehat{\nu}_{B}(t \sin (\theta))\right|^{2} d \theta \leq \int\left|\widehat{\nu}_{B}(t u)\right|^{2} \widehat{\psi}(u / \delta) d u
$$

with an appropriately chosen cut-off function $\psi$. This expression equals

$$
\delta \iint \psi(\delta t(u-v)) d \nu_{B}(u) d \nu_{B}(v) \leq C \delta^{1-\alpha} \cdot t^{-\alpha}
$$

Choosing $\delta=t^{-\gamma_{0}}$, where $\gamma_{0}$ is a small positive number to be determined later, we see that the expression in (3.1) is

$$
\leq C t^{-\gamma_{0}(1-\alpha)} \cdot t^{-\alpha}
$$

We can deal with the neighborhood near $\frac{\pi}{2}$ in the same way, so we omit this part of the calculation.

Now consider

$$
\int_{I}\left|\widehat{\nu}_{A}(t \cos (\theta))\right|^{2}\left|\widehat{\nu}_{B}(t \sin (\theta))\right|^{2} d \theta
$$

where $I$ is an interval that excludes both $\left(0, t^{-\gamma_{0}}\right)$ and a fixed neighborhood of $\frac{\pi}{2}$. By Cauchy-Schwartz, this expression (3.3) is bounded by

$$
C\left(\int_{I}\left|\widehat{\nu}_{A}(t \cos (\theta))\right|^{4} d \theta\right)^{\frac{1}{2}} \cdot\left(\int_{I}\left|\widehat{\nu}_{B}(t \sin (\theta))\right|^{4} d \theta\right)^{\frac{1}{2}} .
$$

Making the change of variables $u=\cos (\theta)$ and $u=\sin (\theta)$, respectively, we see that this expression is

$$
\leq C t^{\gamma_{0}}\left(\int\left|\widehat{\nu}_{A}(t u)\right|^{4} \widehat{\psi}(u) d u\right)^{\frac{1}{2}} \cdot\left(\int\left|\widehat{\nu}_{B}(t u)\right|^{4} \widehat{\psi}(u) d u\right)^{\frac{1}{2}}=C t^{\gamma_{0}} \sqrt{I} \cdot \sqrt{I I},
$$

where $\psi$ is a smooth positive function whose Fourier transform has compact support.

Expanding each expression and changing the order of integration, we obtain

$$
\begin{aligned}
I & =\iiint \int \psi\left(t\left(u_{1}-u_{2}+u_{3}-u_{4}\right)\right) d \nu_{A}\left(u_{1}\right) d \nu_{A}\left(u_{2}\right) d \nu_{A}\left(u_{3}\right) d \nu_{A}\left(u_{4}\right) \\
& \lesssim \nu_{A} \times \nu_{A} \times \nu_{A} \times \nu_{A}\left\{\left(u_{1}, u_{2}, u_{3}, u_{4}\right) \in A^{4}:\left|\left(u_{1}+u_{2}\right)-\left(u_{3}+u_{4}\right)\right|<t^{-1}\right\}
\end{aligned}
$$

and

$$
\begin{aligned}
I I & =\iiint \int \psi\left(t\left(u_{1}-u_{2}+u_{3}-u_{4}\right)\right) d \nu_{B}\left(u_{1}\right) d \nu_{B}\left(u_{2}\right) d \nu_{B}\left(u_{3}\right) d \nu_{B}\left(u_{4}\right) \\
& \lesssim \nu_{B} \times \nu_{B} \times \nu_{B} \times \nu_{B}\left\{\left(u_{1}, u_{2}, u_{3}, u_{4}\right) \in B^{4}:\left|\left(u_{1}+u_{2}\right)-\left(u_{3}+u_{4}\right)\right|<t^{-1}\right\} .
\end{aligned}
$$

Observe that we trivially have

$$
I \lesssim t^{-\alpha} ; \quad I I \lesssim t^{-\alpha}
$$

It follows that

$$
C t^{\frac{\gamma_{0}}{2}} \sqrt{I} \cdot \sqrt{I I} \leq C t^{-\alpha} \leq C t^{\frac{\gamma_{0}}{2}} \cdot t^{-\frac{\operatorname{dim}_{\mathcal{H}}(A \times B)}{2}}
$$


which recovers Wolff's $\frac{4}{3}$ exponent as $\gamma_{0} \rightarrow 0$. Moreover, the only way this estimate does not beat $\frac{4}{3}$ is if

$$
I, I I \geq C t^{-\alpha+\frac{\gamma_{0}}{2}}
$$

for a sequence of $t$ 's going to infinity. The following theorem due to Dyatlov and Zahl ([1]) shows that this cannot happen for Ahfors-David regular sets.

Definition 3.1. [1, Dyatlov and Zahl] Let $X \subset[0,1]^{d}$ and $\nu$ be an outer measure on $X$ with $0<\nu(X)<\infty$. For $r>0$, define the (scale $r$ ) additive energy by

$$
\mathcal{E}(X, \nu, r)=\nu \times \nu \times \nu \times \nu\left\{\left(u_{1}, u_{2}, u_{3}, u_{4}\right) \in X^{4}:\left|\left(u_{1}+u_{2}\right)-\left(u_{3}+u_{4}\right)\right|<r\right\} .
$$

Theorem 3.2. [1, Dyatlov and Zahl] Let $X \subset[0,1]$ be an Ahlfors-David regular set of Hausdorff dimension $\alpha$ and $\nu$ be a measure on $X$ such that for some constant $0<C_{\nu}<\infty$,

$$
C_{\nu}^{-1} r^{\alpha}<\nu(B(x, r))<C_{\nu} r^{\alpha}, \forall x \in X, 0<r<1
$$

Then

$$
\mathcal{E}(X, \nu, r) \leq \widetilde{C} r^{\alpha+\beta_{\nu}}
$$

for some $\beta_{\nu}>0$ and some $\widetilde{C}>0$. In particular, we can choose

$$
\beta_{\nu}=\alpha e^{-\exp \left[K\left(1+\log C_{\nu}\right)^{1 / 2}(1-\alpha)^{-1 / 2}\right]}
$$

where $K$ is an absolute constant; $\widetilde{C}$ depends only on $\alpha$ and $C_{\nu}$.

From Theorem 3.2, Definition 1.2 and the trivial estimate (3.6) of $I I$, it follows that

$$
I \lesssim t^{-\alpha-\beta_{\nu_{A}}}, I I \lesssim t^{-\alpha}
$$

where $\beta_{\nu_{A}}$ is defined in Theorem 3.2. All implicit constants are finite, independent on $t$. Together with the estimate near $\theta=0$ (3.2), we can bound (1.2) by

$$
C t^{-\gamma_{0}(1-\alpha)-\alpha}+C t^{\frac{\gamma_{0}}{2}} t^{-\alpha-\gamma}
$$

where $\gamma=\gamma\left(C_{\nu_{A}}\right)>0$. Let $\gamma_{0}>0$ be a small enough, we get

$$
\int_{S^{1}}|\hat{\mu}(t \xi)|^{2} d \xi \lesssim t^{-\alpha-\delta}
$$

for some $\delta=\delta\left(C_{\nu_{A}}\right)>0$.

\section{Proof of Theorem 1.4}

Let $\nu_{j}$ denote the restriction of the $s_{j}$-dimensional Hausdorff measure to $A_{j}$ and assume without loss of generality that $s_{1} \geq s_{2} \geq \cdots \geq s_{d}$. Parameterize the upper half-sphere in the form

$$
\left\{\left(u_{1}, u_{2}, \ldots, u_{d-1}, \sqrt{1-u_{1}^{2}-\cdots-u_{d-1}^{2}}\right):-1 \leq u_{j} \leq 1\right\} .
$$

Let $\mu$ denote the product measure on $E, \theta_{\omega}$ be the angle between the vector $\omega \in S^{d-1}$ and the hyperplane $\left\{x_{d}=0\right\}$. Without loss of generality, we may assume

$$
\mu\left(\left\{\left(x_{1}, \ldots, x_{d}\right): x_{d}>a\right\}\right), \mu\left(\left\{\left(x_{1}, \ldots, x_{d}\right): x_{d}<a\right\}\right)>0
$$

for some $a \in \mathbf{R}$. An argument identical to the one in the proof of Lemma 2.2 shows the finiteness of

$$
\int_{1}^{\infty}\left(\int_{S^{d-1}}|\widehat{\mu}(t \omega)|^{2}\left|\sin \theta_{\omega}\right| d \omega\right)^{2} t^{d-1} d t
$$


implies that the distance set has positive Lebesgue measure. It follows that

$$
\int_{S^{d-1}}|\widehat{\mu}(t \omega)|^{2}\left|\sin \theta_{\omega}\right| d \omega
$$

is bounded by two terms of the form

$$
\begin{aligned}
& \int_{-1}^{1} \cdots \int_{-1}^{1}\left|\widehat{\nu}_{1}\left(t u_{1}\right)\right|^{2} \cdots\left|\widehat{\nu}_{d-1}\left(t u_{d-1}\right)\right|^{2} \cdot\left|\widehat{\nu}_{d}\left( \pm t \sqrt{1-|u|^{2}}\right)\right|^{2} d u_{1} \cdots d u_{d-1} \\
& \leq \int_{-1}^{1} \cdots \int_{-1}^{1}\left|\widehat{\nu}_{1}\left(t u_{1}\right)\right|^{2} \cdots\left|\widehat{\nu}_{d-1}\left(t u_{d-1}\right)\right|^{2} d u_{1} \cdots d u_{d-1} \cdot
\end{aligned}
$$

By Lemma 2.1, this quantity is

$$
\leq C t^{-s_{1}-\ldots-s_{d-1}} \leq C t^{-s \frac{d-1}{d}}
$$

where $s=\sum_{j=1}^{d} s_{j}$.

Plugging this estimate into the Mattila integral (4.1) we obtain

$$
C \iint|\widehat{\mu}(t \omega)|^{2} t^{d-1} \cdot t^{-s \frac{d-1}{d}} d \omega d t=C \int|\widehat{\mu}(\xi)|^{2}|\xi|^{-s \frac{d-1}{d}} d \xi
$$

and this integral is finite if

which is the case if

$$
d-s<s \frac{d-1}{d}
$$

as desired.

$$
s>\frac{d^{2}}{2 d-1}
$$

\section{References}

[1] Dyatlov, S., and J. Zahl: Spectral gaps, additive energy, and a fractal uncertainty principle. - arXiv:1504.06589, 2015.

[2] ERDoĞAN, B.: A bilinear Fourier extension theorem and applications to the distance set problem. - Int. Math. Res. Not. IMRN 2005:23, 2005, 1411-1425.

[3] Falconer, K.: On the Hausdorff dimensions of distance sets. - Mathematika 32, 1986, 206212.

[4] Greenleaf, A., A. Iosevich, B. Liu, and E. A. Palsson: A group theoretic viewpoint on Erdos-Falconer problems and the Mattila integral. - Rev. Mat. Iberoam. 31:3, 2015, 799-810.

[5] Mattila, P.: Spherical averages of Fourier transforms of measures with finite energy: dimensions of intersections and distance sets. - Mathematika 34, 1987, 207-228.

[6] Mattila, P.: Geometry of sets and measures in Euclidean spaces. - Cambridge Stud. Adv. Math. 44, Cambridge Univ. Press, 1995.

[7] Wolff, T.: Decay of circular means of Fourier transforms of measures. - Int. Math. Res. Not. IMRN 1990:10, 1999, 547-567.

[8] Wolff, T.: Lectures on harmonic analysis. Edited by Izabella Laba and Carol Shubin. - Univ. Lecture Ser. 29, Amer. Math. Soc., Providence, RI, 2003.

Received 31 August 2015 • Revised received 2 November 2015 • Accepted 13 November 2015 